\title{
A Presença da Homeopatia nas Faculdades de Medicina Brasileiras: Resultados de uma Investigação Exploratória
}

\author{
Homeopathy in Brazilian Medical Schools: \\ Results of an Exploratory Investigation
}

Sandra Abrahão Chaim Salles

PALAVRAS-CHAVE

- Homeopatia;

- Medicina;

- Escolas Médicas;

- Educação Médica.

\section{KEY WORDS}

- Homeopathy;

- Medicine;

- Medical Schools;

- Medical Education.

Recebido em: 03/04/2007 Aprovado em: 20/07/2007

REVISTA BRASILEIRA DE EDUCAÇÃO MÉDICA
${ }^{I}$ Faculdade de Medicina da Universidade de São Paulo. Centro de Saúde-Escola Samuel Pessoa, São Paulo, Brasil

${ }^{\text {II }}$ Portaria aprovada pelo Ministério da Saúde em 03 de maio de 2006, publicada em 4 de maio de 2006 aprovando a Política Nacional de Práticas Integrativas e Complementares (PNPIC) no SUS 


\section{INTRODUÇÃO}

Embora seja uma especialidade médica reconhecida pelo Conselho Federal de Medicina desde 1980, a Homeopatia não está presente na maioria das escolas de Medicina, e os fundamentos da prática homeopática não são conhecidos pela grande maioria dos profissionais de saúde. Comparativamente às outras especialidades médicas, esta é uma situação atípica, pois, embora submetida aos mesmos critérios que regulam as demais especialidades, a Homeopatia não dispõe, como elas, do direito de ser apresentada a todos os estudantes de Medicina. Assim, freqüentemente, os médicos iniciam sua prática profissional sem qualquer informação sobre essa outra forma legítima de cuidado médico.

Esse desconhecimento favorece a construção de representações equivocadas e reducionistas sobre a Homeopatia. Nas representações sobre a Homeopatia dos médicos de Vitória, por exemplo, há idéias de misticismo, tratamento natural, tratamento coadjuvante de doenças crônicas, psicossomáticas ${ }^{1}$. Estudantes de Medicina presentes ao $33^{\circ}$ Ecem associam a Homeopatia a efeito placebo, tratamento natural com aspecto místico-religioso, indicado apenas para doenças crônicas ou psicossomáticas, sem fundamentação científica, não reconhecido como especialidade médica ${ }^{2}$. Os homeopatas queixam-se de que a falta de conhecimento sobre sua prática dificulta sua relação com os outros médicos ${ }^{3}$. Foi sugerido que esse desconhecimento sobre as medicinas não convencionais seria uma das razões para os médicos não indagarem ou discutirem com seus pacientes a respeito do uso dessas medicinas ${ }^{4}$. Estudo realizado recentemente com médicos que atendem no SUS indicou que eles desejam saber mais sobre a Homeopatia, para que possam ampliar as possibilidades de encaminhamento de pacientes como uma opção de ajuda diante das limitações terapêuticas que encontram para alguns agravos freqüentes na atenção primária, e também para melhor interagir com os pacientes que fazem uso dessa medicina e acompanhá-los ${ }^{5}$.

Considerando a perspectiva de um incremento significativo da assistência homeopática na rede pública após a definição e publicação da política do Ministério da Saúde para as medicinas não convencionais, cria-se também a iminência de maior contato entre os profissionais homeopatas e não homeopatas, tornando urgente a construção de projetos de educação que divulguem a cultura homeopática de forma mais ampla e sistematizada. Nesse cenário, as faculdades de Medicina constituem um espaço privilegiado para promover essas atividades, pois representam um centro difusor de saberes de reconhecido valor no campo.
As iniciativas dos homeopatas para ampliar seus espaços de assistência, ensino e pesquisa nas universidades têm esbarrado, muitas vezes, em resistências e oposições das instituições e dos profissionais não homeopatas, que têm dificuldades em aceitar uma medicina baseada numa lógica diferente daquela que sustenta a biomedicina. Essas oposições se fundamentam, principalmente, na falta de comprovação da ação das ultradiluições dinamizadas, que são os medicamentos homeopáticos ${ }^{5}$. Os vários estudos de pesquisadores brasileiros e europeus que demonstram a ação das ultradiluições, publicados em revistas cientificas indexadas $^{6-10}$, têm sido insuficientes para remover a resistência, que impede muitos profissionais de se aproximarem da Homeopatia. Para outros, no entanto, a simples observação de resultados obtidos pelo tratamento homeopático em si mesmos, em familiares ou em pacientes foi suficiente para despertar a curiosidade científica e o desejo de investigar o "fato homeopático". Este é um dos fatores que motivou o apoio de alguns docentes/pesquisadores à presença da Homeopatia em algumas faculdades de Medicina do País 5 .

O cenário atual da presença da Homeopatia nas faculdades de Medicina brasileiras é resultado desse embate, com algumas conquistas recentes, como a residência médica em Homeopatia na Unirio, as disciplinas optativas de Homeopatia nos cursos de graduação, o desenvolvimento de algumas pesquisas sobre Homeopatia em ambulatórios e laboratórios de universidades, assim como o financiamento de eventos e pesquisas homeopáticas por órgãos de fomento. Muitas derrotas também ocorreram, e entre elas vale a pena mencionar a perda das faculdades de Medicina homeopáticas que existiram no início do século 20.

O objetivo deste artigo é apresentar um panorama da presença da Homeopatia nas faculdades de Medicina do País nos dias atuais. Ele não se propõe a discutir os elementos que favoreceram a Homeopatia ou, ao contrário, dificultaram sua maior inserção nas escolas médicas, embora este tenha sido um dos principais objetivos do projeto de doutorado do qual este levantamento é parte integrante e inicial.

\section{METODOLOGIA}

O levantamento que se segue foi iniciado com consultas a publicações sobre o tema, ampliado com entrevistas pessoais com homeopatas e finalizado com investigação exploratória das faculdades de Medicina listadas pela Associação Brasileira de Educação Médica (Abem). Certamente, ele não está completo e deverá ser continuamente revisto, pois foi possível perceber a intensa movimentação no campo em relação a esse aspecto. Espera-se, entretanto, por meio deste artigo, colaborar para que os dados sirvam de referência para atualizações periódicas. 


\section{ETAPAS DA INVESTIGAÇÃO}

\section{Levantamento bibliográfico e entrevistas individuais}

Para a pesquisa sobre a presença de atividade homeopática nas faculdades de Medicina do País, inicialmente foi realizada uma revisão dos trabalhos publicados com informações relevantes sobre o assunto. Entre estes, a dissertação de mestrado Homeopatia nas escolas médicas: ensino, assistência e pesquisa no Estado de Säo Paulo ${ }^{11}$ contém informações referentes ao período que vai do reconhecimento da especialidade, em 1980, até 1996. Nesse estudo, foram enviados questionários semi-abertos a 19 faculdades do Estado de São Paulo. Seus resultados apontaram ocorrências localizadas de ensino de Homeopatia, predominando experiências passadas, de perfil teórico e extracurricular.

Outra fonte de informações foi a pesquisa realizada pela Comissão Homeopatia na Graduação, da Associação Médica Homeopática Brasileira $(\mathrm{AMHB})^{12}$, que enviou questionário estruturado a docentes de Homeopatia de faculdades de Medicina sabidamente possuidoras da disciplina de Homeopatia na graduação médica. Os resultados dessa pesquisa, coletados entre 1997 e 2002, informam sobre nove faculdades que responderam os questionários enviados. Por meio dela foi possível identificar as instituições que, em 2002, mantinham atividades de ensino homeopático, oferecidas sob a forma de disciplina optativa ou apresentação de conteúdos de Homeopatia dentro de outras disciplinas. Foram citadas: Universidade Federal de Santa Catarina (UFSC), Universidade Federal da Paraíba (UFPB), Universidade do Rio de Janeiro (Unirio), Faculdade de Medicina da Universidade Federal de Uberlândia (UFU), Faculdade de Medicina da Universidade de São Paulo (FMUSP), Universidade Estadual de Campinas (Unicamp) e Universidade Federal Fluminense (UFF). Além destas, a Universidade de Brasília e a Faculdade Evangélica do Paraná referiram ter oferecido anteriormente a disciplina de Homeopatia.

Outras informações foram obtidas na página eletrônica da $\mathrm{AMHB}^{13}$, que apresenta alguns textos sobre a história da introdução da Homeopatia em algumas faculdades de Medicina, como USP, UFPE e Unifesp.

Entrevistas pessoais com médicos homeopatas, realizadas ao longo dos últimos três anos, durante eventos homeopáticos, forneceram informações sobre atividades homeopáticas ainda não conhecidas. Estas informações foram, então, confirmadas e ampliadas mediante contato direto com as instituições ou docentes responsáveis pelas disciplinas.

\section{Investigação exploratória}

Uma investigação exploratória foi realizada em fevereiro de 2005. Tomando como referência a lista das faculdades de
Medicina da Abem de fevereiro de 2005, foram enviadas mensagens por correio eletrônico a coordenadores e/ou diretores de 101 faculdades de Medicina indagando sobre a existência de atividades homeopáticas nessas instituições. Não foram enviadas mensagens a 14 faculdades sobre as quais já se dispunha de informações recentes e confirmadas. Foram obtidas 32 respostas ao total de mensagens enviadas.

\section{RESULTADOS}

A seguir, apresenta-se o resultado geral obtido nessas investigações. Estão incluídos os dados sobre as faculdades de Medicina que informaram não oferecer atividades em Homeopatia, para que sirvam de referência a outros estudos e futuras atualizações.

Relação das faculdades segundo respostas sobre a presença de atividades homeopáticas (total de $\mathbf{1 1 5}$ faculdades de Medicina do País - Abem 2005)

\section{5 faculdades não oferecem qualquer atividade em Homeopatia}

Escola de Ciências Médicas de Alagoas Curso de Medicina da Universidade Federal de Alagoas Curso de Medicina da Universidade Federal do Piauí Curso de Medicina da Universidade Federal de Sergipe Faculdade de Medicina da Universidade Federal da Bahia Cursos de Medicina da Universidade Federal do Ceará

(Barbalha, Fortaleza e Sobral)

Faculdade de Medicina de Petrópolis

Faculdade de Medicina da Universidade Federal do Rio de Janeiro

Escola de Medicina Unigranrio

Escola de Medicina Souza Marques

Curso Médico do Centro Universitário de Volta Redonda (UniFOA)

Curso de Medicina da Universidade Federal do Espírito Santo Faculdade de Medicina da Unipac - Juiz de Fora

Faculdade de Medicina da Universidade Federal de Juiz de Fora Faculdade de Ciências Médicas de Minas Gerais

Faculdade de Ciências Médicas da PUC-SP

Faculdade de Medicina da USP - Ribeirão Preto - SP

Faculdade de Medicina da Unoeste - Presidente Prudente

Faculdade de Medicina de Catanduva

Curso Médico da Universidade Estadual de Maringá

Curso de Medicina da Unioeste

Curso de Medicina da Universidade Estadual de Londrina

Faculdade de Medicina Universidade de Passo Fundo

Curso de Medicina da Universidade de Caxias do Sul

Faculdade de Medicina da PUC 
Curso de Medicina da Universidade Federal de Santa Maria Curso de Medicina da Universidade Federal de Rondônia Faculdade de Medicina da Universidade de Cuiabá

Faculdade de Medicina da UFMS - Dourados

Faculdade de Ciências Médicas da Santa Casa de SP

Faculdade de Ciências Médicas da PUC - Campinas

Curso de Medicina da Universidade Federal de Santa Catarina

Faculdade de Ciências da Saúde da Universidade Federal do

Amazonas

\section{7 faculdades oferecem atividades relacionadas à Homeo- patia}

Faculdade de Medicina da UFF

Escola de Medicina e Cirurgia da Unirio

Faculdade de Medicina do ABC

Faculdade de Medicina da USP

Curso de Medicina da Unifesp

Faculdade de Medicina de Jundiaí

Faculdade de Ciências Médicas da Unicamp

Curso de Medicina da Universidade de Mogi das Cruzes

Curso de Medicina da Unaerp

Curso de Medicina da Fundação Universidade Regional de Blumenau

Faculdade Evangélica do Paraná

Curso de Medicina da UEA

Curso de Medicina da UFPB

Curso de Medicina da UFPE

Faculdade de Medicina da UFRN

Curso de Medicina da UFU

Faculdade de Medicina da UnB

Informações sobre as atividades desenvolvidas em cada instituição

Breve histórico sobre as atividades homeopáticas nas instituições

\section{Escola de Medicina e Cirurgia da Unirio}

É a única faculdade de Medicina que tem a Homeopatia entre suas disciplinas obrigatórias, e seu hospital-escola é o único a oferecer residência médica em Homeopatia. A história da Homeopatia nessa instituição se inicia com a criação da Faculdade de Medicina Homeopática do Rio de Janeiro, em abril de 1912, com base na lei Rivadavia Correia, que dava aos estabelecimentos de ensino autonomia didática e administrativa. Divergências internas e críticas do Instituto Hahnemanniano à organização do ensino nessa instituição geraram ações judiciais que culminaram com sua extinção e a criação de uma nova faculdade, como órgão de ensino do Instituto e denominada Faculdade Hahnemanniana. Esta faculdade congregava cadeiras de Homeopatia e alopatia, ensinando filosofia e terapêutica homeopática, assim como disciplinas de Anatomia, Fisiologia, Patologia e Clínica. Apenas em 1921 ela conseguiu a equiparação dos direitos de seus formados aos de formados pelas outras faculdades de Medicina do País. Constantemente ameaçada pelo Conselho Nacional de Ensino de perder o direito à equiparação, em 1938 as cadeiras de Homeopatia tornaram-se facultativas, e em 1948 ela se tornou independente do Instituto Hahnemanniano, passando a se chamar Escola de Medicina e Cirurgia do Rio de Janeiro ${ }^{14}$.

Desde aquela época, o ensino da Homeopatia foi mantido, por intermédio de um departamento próprio, atualmente denominado Departamento de Homeopatia e Terapêutica Complementar, responsável por cinco disciplinas - uma obrigatória (Matéria Médica Homeopática) e três optativas (Terapêutica Homeopática, Clínica Homeopática I e Clínica Homeopática II).

Em 2003, após trabalho de planejamento e sensibilização da administração do hospital universitário Gaffré Guinle, promovido pelo presidente da Comissão de Residência Médica desse hospital, Dr. Rossano Kepler, e pelo diretor do Instituto Hahnemanniano do Brasil, Dr. Francisco Freitas, foi aprova-

\section{QUADRO 1}

Faculdades de Medicina segundo o tipo de atividade homeopática desenvolvida em 2005

\begin{tabular}{|c|c|c|c|c|c|c|}
\hline $\begin{array}{l}\text { Residência } \\
\text { médica e } \\
\text { disciplinas } \\
\text { obrigatórias }\end{array}$ & $\begin{array}{c}\text { Disciplina } \\
\text { optativa, } \\
\text { ambulatório e } \\
\text { pesquisa }\end{array}$ & $\begin{array}{c}\text { Disciplina } \\
\text { optativa e } \\
\text { ambulatório }\end{array}$ & $\begin{array}{l}\text { Disciplina } \\
\text { optativa }\end{array}$ & $\begin{array}{c}\text { Conteúdos em } \\
\text { outras disciplinas }\end{array}$ & $\begin{array}{c}\text { Liga de } \\
\text { Homeopatia }\end{array}$ & $\begin{array}{c}\text { Curso de } \\
\text { especialização em } \\
\text { Homeopatia }\end{array}$ \\
\hline \multirow[t]{5}{*}{ Unirio } & USP & UFPB & UFU & UnB & Unicamp & FMJ \\
\hline & Unifesp & UFPE & UFRN & Unaerp & Unaerp & \\
\hline & UFF & UMC & UEA & & Unifesp & \\
\hline & & FEPAR & Furb (Blumenau) & & & \\
\hline & & & FM ABC & & & \\
\hline
\end{tabular}


da a primeira residência médica em Homeopatia do País. De entrada direta e com duração de dois anos, as atividades de ensino homeopático são desenvolvidas em ambulatórios e enfermarias do hospital.

\section{Curso de Medicina da Universidade Federal de São Paulo}

Nesta instituição, a primeira atividade homeopática foi um curso realizado, em 1955, na então Escola Paulista de Medicina (EPM), patrocinado pelo Centro Acadêmico, pela Associação Paulista de Homeopatia e pela Federação Brasileira de Homeopatia. Em 1997, o Departamento de Medicina aprovou a criação de uma comissão de pesquisadores da universidade e médicos homeopatas convidados, atendendo à solicitação de um ministro da Saúde ao reitor para que a universidade avaliasse a eficácia das medicinas não ortodoxas. Desde então, foram conduzidas algumas pesquisas (ensaios clínicos e estudos experimentais) nos Departamentos de Geriatria e Otorrinolaringologia, sob a orientação de pesquisadores não homeopatas. No Departamento de Nefrologia, um trabalho experimental sobre o uso da Homeopatia em infecções urinárias em ratas foi apresentado como dissertação de mestrado ${ }^{15}$.

Desde 2001, uma disciplina eletiva, Introdução à Homeopatia, tem sido oferecida aos alunos de Medicina da Unifesp, com carga de 12 horas. Foram criados setores de Homeopatia nas disciplinas de Geriatria e Clínica Médica, assim como a Liga Acadêmica de Homeopatia, que oferece atendimento ambulatorial a funcionários da instituição.

\section{Faculdade de Medicina da Universidade de São Paulo}

Em 2002, a Comissão de Graduação aprovou a disciplina Fundamentos de Homeopatia, ministrada desde então aos alunos do quarto ano. Ela é oferecida duas vezes ao ano, com carga horária semestral de 75 horas-aula, compreendendo aulas teóricas, prática em ambulatório, auto-experimentação patogenética e seminários. O conteúdo programático segue o modelo sugerido pela AMHB. A aprovação dessa disciplina sucedeu a um trabalho de sensibilização de docentes da faculdade realizado por um ex-aluno da instituição que, em 1999, iniciou um ensaio clínico para avaliação da eficácia e efetividade de tratamento homeopático no Departamento de Clínica Médica.

Trabalhos nas áreas de pesquisa básica, clínica, institucional e social têm sido desenvolvidos paralelamente. Em 1989, um docente do Departamento de Medicina Preventiva desenvolveu uma pesquisa sobre os princípios homeopáticos ${ }^{16}$; em 1998, foi apresentada uma dissertação de mestrado sobre a medicina homeopática ${ }^{17}$; entre 2004 e 2006, foram apresentadas nesse departamento três outras teses sobre práticas alternativas em saúde ${ }^{5,18,19}$.
Uma tese de doutorado baseada em pesquisa experimental e que demonstra a ação das ultradiluições foi desenvolvida e apresentada no Departamento de Patologia, com resultados publicados em revista internacional ${ }^{9}$. Em 2005, foi publicado um artigo sobre estudo realizado com alunos da graduação da FMUSP, indicando sua atitude de grande interesse no aprendizado dos fundamentos da Homeopatia e acupuntura, e de defesa da incorporação dessas modalidades de assistência aos serviços públicos de saúde ${ }^{20}$.

\section{Faculdade de Medicina da Universidade Federal Fluminense}

Desde 1996, o Departamento de Saúde e Sociedade, do Instituto de Saúde da Comunidade, oferece disciplinas optativas no curso de Medicina: Introdução à Homeopatia, Propedêutica Homeopática e Terapêutica Homeopática. Ela também é abordada em duas disciplinas obrigatórias para os alunos de Medicina: Paradigmas Médicos e Medicina Empírica I - a Medicina Homeopática.

Oferece atendimento homeopático no Hospital Antonio Pedro, com fornecimento de medicamentos aviados no Laboratório de Homeopatia e Fitoterapia da própria faculdade. Nessa instituição, foi realizado junto à nefrologia um ensaio clínico (randomizado, duplo-cego, placebo-controlado) que demonstrou a eficácia do tratamento homeopático para reduzir o prurido de pacientes renais crônicos em hemodiálise ${ }^{10}$.

\section{Curso de Medicina da Universidade Federal de Uberlândia}

A chegada de um médico homeopata à instituição na década de 1980 se somou às perspectivas de mudança em função de uma reforma curricular que estava em andamento, desencadeando um movimento de inserção da Homeopatia que teve início com cursos de extensão. A partir de 1984, a faculdade de Medicina passou a oferecer aos alunos da graduação uma disciplina optativa: Introdução à Homeopatia. Durante certo período, manteve, em seu hospital-escola, a possibilidade do uso de terapêutica homeopática associada à terapêutica convencional, prescrita por médicos do Departamento de Clínica Médica com formação em Homeopatia. Promoveu, ainda, um curso de formação de docentes em Homeopatia e um concurso público para docente de Homeopatia na disciplina de Clínica Médica. Atualmente, a única atividade relacionada à especialidade, de caráter estritamente teórico, é a disciplina optativa de Homeopatia, com pouca repercussão entre os outros profissionais da faculdade.

\section{Faculdade de Ciências Médicas da Unicamp}

Desde 1999, foi organizado um grupo de estudos e atendimento homeopático que se vinculou à Liga Estudantil, consti- 
tuindo atualmente a Liga Homeopática. Oferece aos alunos do segundo ano uma disciplina optativa, além de atendimento clínico didático. Foi apresentado um projeto para ambulatório de Homeopatia no Departamento de Clínica Médica, rejeitado por seus membros.

\section{Curso de Medicina da Universidade Federal da Paraíba}

A disciplina Fundamentos da Homeopatia, optativa, foi criada em 1983 por iniciativa de Virginia Siqueira, professora da disciplina de Microbiologia do Departamento de Fisiologia e Patologia, atendendo também à demanda de alunos da graduação que participavam de um grupo de estudos sobre Homeopatia criado em 1979. A disciplina foi oferecida inicialmente apenas aos alunos de Medicina e, desde 1984 até hoje, também aos alunos de Enfermagem, Farmácia, Fisioterapia, Nutrição e Odontologia. É vinculada ao Departamento de Fisiologia e Patologia do Centro de Ciências da Saúde.

A carga é de três horas semanais, totalizando 45 horas semestrais. São seis turmas por semestre, somando cerca de 300 alunos/ano. Vem sendo oferecida ininterruptamente há 21 anos. Foram aprovados 5.388 alunos na disciplina de 1984 a 2003, segundo planilha do Departamento, dos quais 1.840 alunos do curso de Medicina.

Desde 1991, há também atendimento homeopático no ambulatório de Pediatria do Hospital Universitário. Em 1995, foi criado o ambulatório de Homeopatia no mesmo hospital, onde atendem atualmente cinco médicos, em um turno por semana.

\section{Curso de Medicina da Universidade Federal de Pernambuco}

Há alguns anos, mantém um ambulatório didático de Homeopatia no Setor de Pediatria do Hospital das Clínicas, sob a orientação da professora Odimariles Dantas. Em 2004, foi aprovada a disciplina eletiva de Homeopatia, com 30 horas teóricas e 30 horas de prática ambulatorial, que começou a ser oferecida aos alunos em 2005. A instituição oferece residência médica em acupuntura, cujas atividades didáticas são realizadas no Hospital de Clínicas.

\section{Faculdade de Medicina de Jundiaí}

Um grupo de homeopatas que atendia no Centro de Saúde Pinheiros (São Paulo) propôs um convênio com a faculdade para implantar um curso de pós-graduação em Homeopatia. A proposta foi aprovada em dezembro de 2002, por unanimidade, na Congregação da faculdade, e o curso teve início em agosto de 2003. A Secretaria Municipal de Saúde indicou $\mathrm{o}$ atendimento prestado na instituição como referência para o SUS.
Curso de Medicina da Universidade do Estado do Amazonas

A partir de 2003, a disciplina Homeopatia, optativa, passou a ser oferecida uma vez por ano aos alunos de graduação em Medicina, Enfermagem e Odontologia. Com carga de 30 horas, é oferecida aos alunos do ciclo clínico (sétimo período em diante). Com conteúdo teórico, de caráter informativo e introdutório, não dispõe de ambulatório.

\section{Curso de Medicina da Universidade Federal do Rio Grande do Norte}

Oferece a disciplina Introdução à Homeopatia, ligada ao Departamento de Saúde Coletiva, com carga de 30 horas.

\section{Curso de Medicina da Fundação Universidade Regional de Blumenau}

Desde 2004, oferece semestralmente a disciplina optativa de Homeopatia, com 36 horas-aula.

\section{Faculdade de Medicina do ABC}

Oferece disciplina optativa.

\section{Faculdade Evangélica do Paraná}

Oferece disciplina optativa de Homeopatia desde 2002, com 56 horas-aula, aos alunos do quinto ano. Dispõe de ambulatório assistencial.

\section{Curso de Medicina da Universidade de Mogi das Cruzes}

Desde 2004, os alunos do internato freqüentam o ambulatório de Homeopatia durante o rodízio na Clínica Médica. Desde 2005, oferece uma disciplina de Homeopatia aos alunos do quarto ano, com 32 horas-aula.

\section{Algumas ocorrências passadas}

A apresentação destes dados revela a fragilidade de algumas conquistas que não se mantiveram nas universidades. Esses espaços foram abertos por iniciativa pessoal de médicos homeopatas e, muitas vezes, ficaram na dependência desse profissional para garantir sua continuidade, tendo se encerrado quando o profissional se afastou da instituição. Tais casos são exemplos atuais de um processo que tem ocorrido em locais diferentes, denotando, entre outros aspectos, as dificuldades na articulação política dos homeopatas, que não conseguiram obter legitimação e garantias institucionais para suas conquistas pessoais.

\section{Faculdade de Ciências Médicas da PUC de Campinas}

Durante alguns anos, manteve atendimento homeopático em seu Centro de Saúde Escola, onde docentes de Medicina Social e Preventiva apresentavam fundamentos de Homeopatia, 
vinculados à prática. Esta forma de inserção do ensino homeopático permitia apresentar a Homeopatia "como um saber médico com racionalidade e modelo científico diferentes da medicina convencional e como recurso tecnológico adequado à atenção primária à saúde" ${ }^{\prime \prime}$. Esta atividade foi interrompida desde 2003, segundo entrevista com a diretora do Centro de Saúde, por mudança da docente responsável para outro Estado.

\section{Faculdade de Ciências da Saúde da Universidade Federal do Amazonas}

Já ofereceu a disciplina optativa de Introdução à Homeopatia aos alunos dos cursos de Farmácia e Medicina, mas essas atividades se encerraram em 1990.

\section{Curso de Medicina da Universidade Federal de Santa Catarina}

Desde o segundo semestre de 1998, os alunos do décimo período de Medicina, durante o internato em Saúde Coletiva, entraram em contato com conteúdos sobre os princípios básicos da Homeopatia, ministrados em quatro horas mensais. $\mathrm{Na}$ pós-graduação, o curso de especialização multiprofissional em Saúde da Família oferecia a disciplina Métodos Terapêuticos Complementares, que abordava temas da Homeopatia. Esta atividade didática foi interrompida em 2005, pois o docente responsável se mudou para outro Estado.

\section{Faculdade de Ciências Médicas da Santa Casa de São Paulo}

Em 1967, esta faculdade realizou um curso de Homeopatia, que promoveu a retomada dessa especialidade no Estado. Em 1977, a instituição sediou a IV Jornada Paulista de Homeopatia e alguns cursos informativos para estudantes de Medicina. Por iniciativa de seu provedor, Dr. Mario Altenfelder Silva, foi criado o ambulatório de Homeopatia da Santa Casa de Misericórdia de São Paulo, em abril de 1988, que se propôs a promover a convivência entre terapêuticas diferentes num mesmo espaço físico. Este ambulatório funcionou de maneira regular por seis anos, prestando atendimento e promovendo, por meio de intercâmbio com a prefeitura, cursos de reciclagem para médicos homeopatas da rede, avalizados pelo Departamento de Clínica Médica. Suas atividades foram encerradas em 1993 por iniciativa da diretoria clínica, colocando a instituição como um dos exemplos de retrocesso no movimento de ocupação de espaços acadêmicos.

\section{CONCLUSÃO}

As faculdades de Medicina ainda representam um espaço do campo da saúde que evidencia a condição de saber não hegemônico da Homeopatia. A presença da Homeopa- tia nessas instituições é pouco freqüente e ocorre de maneira diversificada, dependendo sempre de iniciativas pessoais de homeopatas. Em geral de caráter eletivo, as disciplinas ficam a cargo desses profissionais, que, por não serem concursados, não obtêm a garantia institucional de continuidade da atividade homeopática após seu afastamento. Essa situação implica, freqüentemente, a perda de espaços conquistados. Constitui exceção a situação da Homeopatia na Unirio, que, além das disciplinas obrigatórias que já oferecia aos alunos, implantou a primeira residência médica em Homeopatia do País.

A produção científica dos homeopatas que estão nas universidades, ainda que reduzida, é bastante significativa, demonstrando os resultados do trabalho conjunto entre homeopatas e outros pesquisadores da saúde.

Acredita-se que, diante do incremento da demanda social por essa forma de cuidado em saúde e das disposições da Portaria 971, publicada pelo Ministério da Saúde e que recomenda a implementação da assistência homeopática no âmbito do SUS, seja necessário criar núcleos de ensino e informação sobre a Homeopatia nessas instituições. Cabe aos homeopatas incentivar a criação desses núcleos e buscar a regulamentação dessas atividades, garantindo que elas sejam capazes de esclarecer seus pares profissionais sobre os procedimentos homeopáticos e seus fundamentos. E que também sejam pólos receptivos de outros saberes, de forma a contribuir com a revitalização da cultura homeopática.

\section{AGRADECIMENTOS}

Agradeço aos colegas Anna Alice M. Schroeder, Ana Marta Cavalcanti, Climério A. Figueiredo, Fernando Bragança, Fernando Bignardi, Francisco Freitas, Graciela Pagliaro, Mario C. Sposatti, Miltom Lopes e Odimariles Dantas, pelas informações e documentos fornecidos.

\section{REFERÊNCIAS}

1. Mageste RE Machado VL, Novaes AR, Figueiredo TM, Representações da homeopatia entre médicos alopatas de Vitória ES. In: Anais do XXIV Congresso Brasileiro de Homeopatia; 1998 out. 29-nov. 2; Gramado, Brasil.

2. Teixera MZ. Homeopatia, desinformação e preconceito. In: Anais do VIII Encontro Internacional de Pesquisas Institucionais em Homeopatia; 2004 São Paulo, Brasil.

3. Salles S A C. O perfil domédico homeopata São Paulo; 2001. Mestrado [dissertação] — Faculdade de Saúde Pública da Universidade de São Paulo

4. Eisenberg DM et al. Unconventional medicine in the United States: prevalence, costs and pattern of use. N Eng J Med 1993;328:246-52. 
5. Salles SAC. Interface entre a Homeopatia e a Biomedicina: o ponto de vista dos profissionais de saúde não homeopatas São Paulo; 2006. Doutorado [Tese] - Faculdade de Medicina da Universidade de São Paulo.

6. Zacharias CR. Physical research in dynamized systems. Méd Hypotheses. Jun 2002; 58(6):523-6

7. Belon P, Cumps J, Ennis M, Mannaioni PF, Saint-Laudy J, ROberfroid M, Wiegant FAC. Inhibition of human basophil degranulation by successive histamine dilutions: results of a European multi-centre trial, inflamm. Res 1999: 48 (Suppl 1); $17-8$.

8. Belon P, Cumps J, Ennis M, Mannaioni PF, Roberfroid M, Saint-Laudy J, Wiegant FAC. Histamine dilutions modulate basophil activation, Inflamm res. 2004; 53(5):181-8

9. Guedes JR, Ferreira CM, Guimarães HM, Saldiva PHN, Capelozzi VL. Homeopathically prepared dilution of Rana catesbiana Thyroid glands modifies its rate of metamorphosis. Homeopathy 2004; 93:132-7.

10. Cavalcanti AMS, Rocha LM, Carillo JrR, Lima LUO, Lugon JR. Effects of homeopathic treatment on pruritus of haemodialysis patients: a randomised placebo-controlled double-blind trial. 2003; 92(4): 177-81.

11. Gianesella EMF. Homeopatia nas escolas médicas: ensino, assitência e pesquisa no estado de São Paulo. São Paulo; 1998. Mestrado [dissertação] — Faculdade de Saúde Pública da Universidade de São Paulo.

12. Associação Médica Homeopática Brasileira. Comissão Homeopatia na Graduação. homeopatia na graduação médica. Rio de Janeiro; 2003. (Relatório)

13. Associação Médica Homeopática Brasileira [página eletrônica] Minas Gerais, Brasil; 2003. [capturado 18 mar. 2004]. Disponível em: http: / /www.amhb.org.br.

14. Fundação Oswaldo Cruz. Casa de Oswaldo Cruz. Dicionário histórico-biográfico das Ciências da Saúde no Brasil
(1832-1930). [página eletrônica]. Riode Janeiro, Brasil; [capturado 6 set. 2005]. Disponível em: http:/ /www.dichistoriasaude.coc.fiocruz.br.

15. Gonçalves MI. O uso da homeopatia no tratamento da infecção urinária em ratas. São Paulo; 2001. Doutorado [Tese] - Faculdade de Medicina da Unifesp.

16. Novaes RL. O tempo e a ordem: sobre a homeopatia. São Paulo: Cortez/Abrasco; 1989

17. Rosenbaum P. A homeopatia como medicina do sujeito: raízes históricas e fronteiras epistemológicas. São Paulo; 1998. Mestrado [dissertação] — Faculdade de Medicina da Universidade de São Paulo.

18. Akyiama K. Práticas não-convencionais em medicina no Município de São Paulo. São Paulo;2004. Doutorado [Tese] - Faculdade de Medicina da Universidade de São Paulo.

19. Rosenbaum P. Entre arte e ciência: fundamentos hermenêuticos da homeopatia como medicina do sujeito. São Paulo; 2005. Doutorado [Tese] — Faculdade de Medicina da Universidade de São Paulo.

20. Teixeira MZ, Chin AL, Martins MA O ensino da homeopatia e da acupuntura na Faculdade de Medicina da Universidade de São Paulo: atitude dos acadêmicos. Med. J 2005; 123(2):77-82.

\section{CONFLITOS DE INTERESSE}

Declarou não haver

\section{ENDEREÇO PARA CORRESPONDÊNCIA}

Rua Tomé de Souza, 130

Lapa - São Paulo

CEP. 05079-000 / SP

E-mail: sandrachaim@terra.com.br 